\title{
The Superiority of the Anterior Transposition Surgical Method to Decompression Proceduresin a Case of Secondary Cubital Entrapment Neuropathy
}

\author{
Ertuğrul Allahverdi ${ }^{1}$, Tülay Diken Allahverdi ${ }^{2}$ \\ ${ }^{1}$ Kafkas University Department of Orthopedics and Traumatology, Kars, Turkey \\ ${ }^{2}$ Kafkas University Department of General Surgery, Kars, Turkey \\ Received: 13 September 2016, Accepted: 01 December 2016, Published online 25 April 2016 \\ (C) Ordu University Institute of Health Sciences, Turkey, 2017
}

\begin{abstract}
Cubital Entrapment Neuropathy (cubital tunnel syndrome) is a peripheral entrapment neuropathy coming right after the carpal tunnel syndrome in terms of frequency. It is twice as common in adult males as in females. We report our surgical experience with the anterior transposition method. A 41-year-old male patient presented to our clinic with symptoms of functional weakness of the right hand hypothenar region and 3rd- 4th intrinsic lumbrical muscles together with atrophy and claw finger symptom in the 4th and 5th fingers (Grade III) that had been increasing for the last 19 months. The patient underwent ulnar nerve decompression. There was no improvement 5 months after the surgery and anterior transposition of the ulnar nerve was therefore performed as secondary surgery. No complication occurred afterwards.The anterior transposition method used in our case with secondary cubital tunnel syndrome has a high success rate when performed properly although it is a more invasive intervention. While the only accepted treatment for idiopathic cubital tunnel syndrome is decompression, the anterior transposition method can be used for posttraumatic secondary cubital tunnel syndrome. Surgical Methods of Anterior Transposition for Secondary Cubital Entrapment vs. Decompression Procedures.
\end{abstract}

Key words: Cubital tunnel syndrome, Anterior intramuscular transposition, decompression, Ulnar nerve

Address for correspondence/reprints:

Ertuğrul Allahverdi

Telephone number: +90 (506) 6319651

E-mail: ertugrulallahverdi@hotmail.com

DOI: 10.19127/mbsjohs.309377

\section{Introduction}

Cubital tunnel syndrome is a peripheral entrapment neuropathy where the sensory or motor part or both parts of the ulnar nerve are affected. It is the second most common peripheral entrapment neuropathy following the carpal tunnel syndrome (Keineret al.,2009; Flores, 2010; Anderton and Webb, 2010). It is twice as common in adult men as in women. The etiology includes past fractures, luxation's, cubits valgus deformity, contractures in the elbow area, high body mass index, alcohol, smoking addiction, increased duration and frequency of mobile phone use (mobile phone elbow), weight holding-catching, work involving lifting weights with the elbow in flexion over the long term, diabetes, thyroid diseases, renal failure, inflammatory arthropathy, pregnancy, malnutrition, and tumoral masses (ganglion, lipoma, hemangioma 
etc.). However, many cases are idiopathic (Dhia andJaddue,2009; Keiner et al.,2009; Andertonand Webb, 2010). Thoracic outlet syndrome, C8 / Th 1 radiculopathy (cervical rod compression), brachial plexus pathologies, pathologies in Guyon's canal at the wrist (artery aneurysm, venous thromboses, Villon nodular synovitis, etc.) ulnar nerve entrapment, double crush syndrome, and Pancoast tumor should be considered and investigated in the differential diagnosis (Uysal et al.,2007; Dhia and Jaddue,2009; Anderton and Webb,2010).

The main symptom can be pain. The symptoms can start insidiously and can be temporary at the beginning. The pain occurs in the hypothenar section of the hand, little finger, and ulnar half of the ring finger at the early period. Disruption in finger functions, dropping objects, difficulties in catching and squeezing objects occur if the compression continues or increases in severity. The Wartenberg symptom can be seen in advanced cases where symptoms are marked. The Froment test, Tinel percussion test, elbow flexion test, two-point differentiation, and the pinprick test are used for the diagnosis. The Bishop evaluation is also conducted. In addition to the specific tests mentioned above, MR, EMG and high-resolution ultrasonography are used for clinical research and evaluations (Kutlay et al.,2009; Schnablet al.,2010; Ibaet al.,2010; Thatte and Mansukhani,2011; Kılıç and Özçakar,2011). While conservative treatment protocols are primarily used in the treatment of patients with mild cubital tunnel syndrome, surgical procedures are preferred in advanced severe cases. In general, only ulnar nerve interior tunnel decompression is implemented in patients with idiopathic cubital tunnel syndrome and anterior transposition (subcutaneous, intramuscular, submuscular).
The endoscopic in situ cubital tunnel relaxationdecompression or medial epicondylectomy methods are used in severe cases with a history of trauma (Asamoto et al.,2005; Keineret al.,2009;Dhia and Jaddue, 2009; Sreedbaran et al,2010; Tubbs et al.,2010).

\section{Case Report}

A 41-year-old male patient presented to our clinic with symptoms of numbness in the right hand hypothenar area, pain in the medial epicondyle area and elbow movement restriction for the last 21 months. The personal history revealed that he was exposed to the interventions of a bonesetter and osteopath after a supraepicondylarhumerus fracture at the age of 7 . Flexion contracture and cubitus valgus deformity of the right elbow had developed afterwards. No vascular, neurologic, or muscular function loss was present except for mild valgus disorder and extension restriction at the elbow during childhood and puberty. There was right elbow medial epicondyle pain, positive signs on the Tinel percussion test and Froment test, positive Wartenberg symptom, 14 degrees of cubitus valgus deformity, and 20 degrees of flexion contracture of the right elbow on the physical and radiological examination MR revealed no pathology of the humerus, forearm or wrist, but there was medial epicondyle pseudoarthrosis at the elbow and edema around the ulnar nerve at the cubital tunnel. The patient was started on oral NSAIDs, flexion restriction, and oral $\mathrm{B} 1, \mathrm{~B} 6$, and $\mathrm{B} 12$ vitamins as conservative treatment. In the meantime, he was directed to the neurology clinic. Two separate controlled EMG evaluations revealed severe cubital tunnel syndrome. Guyon canal neuropathy was not found. No pathology was found in cranial, cerebrovascular, cervical vertebrae and nerve roots or the brachial plexus on cervical and cranial MRI. No pathology was observed in the chest graph. According to the classification of McGowan and Dellon, the cubital syndrome was Grade III (severe, advanced) in our case (Figure 1 and 2). 


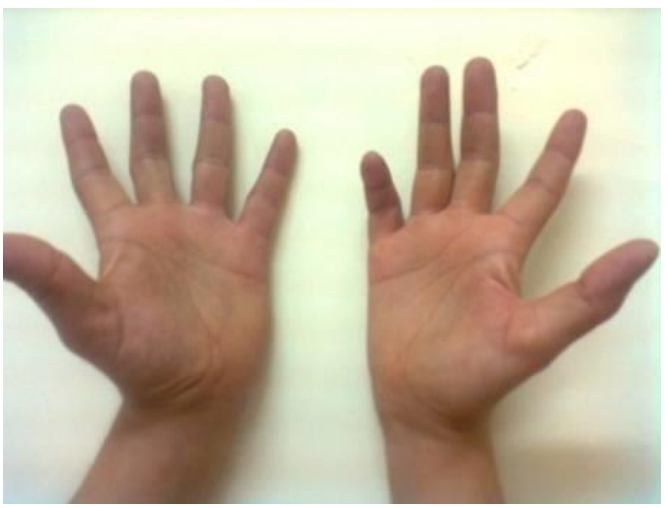

Figure1: Right hand hypothenary atrophy, thumb flexion status in 5th, 4th and 3rd fingers (pre op.)

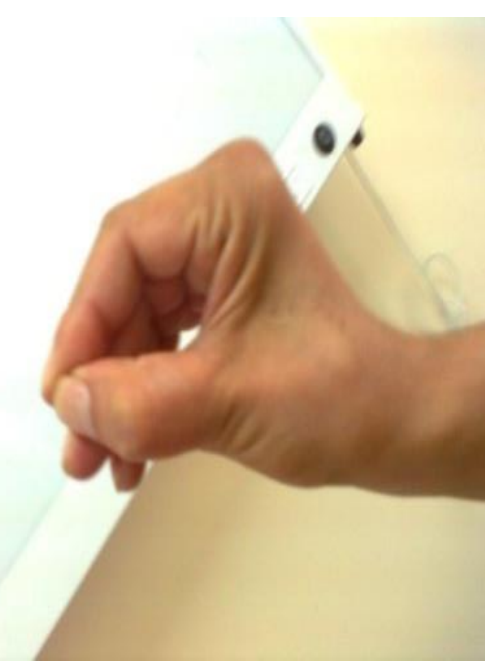

Figure 2: Froment sign; Hyperflexion of the in the holding, catching position (pre op.).



Figure 3: Intro op Ulnar nerve cubital tunnel during decompression

Only ulnar nerve decompression was performed during the initial surgical intervention (Figure 3 and 4)

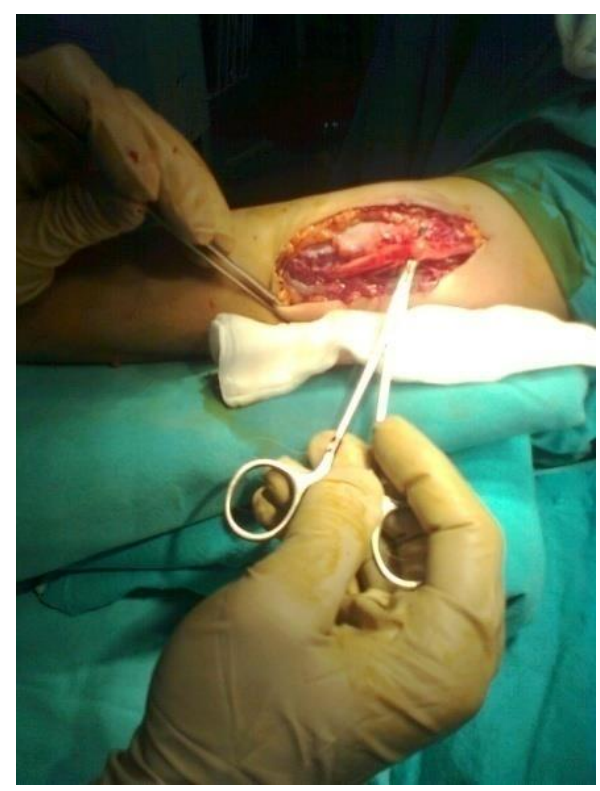

Figure 4: Intro op final state of cubital tunnelat and ulnar nerve at decompression

Minor improvement was obtained in hypothenar sensation at the post-operative 3rd and 5th months. However, the cubital tunnel syndrome continued in EMG repeated after the post-operative 3rd month. Revision surgery was scheduled as the symptoms of the patient continued during work and social activities. Anterior transposition was performed as revision surgery (Figure 5,6,7).

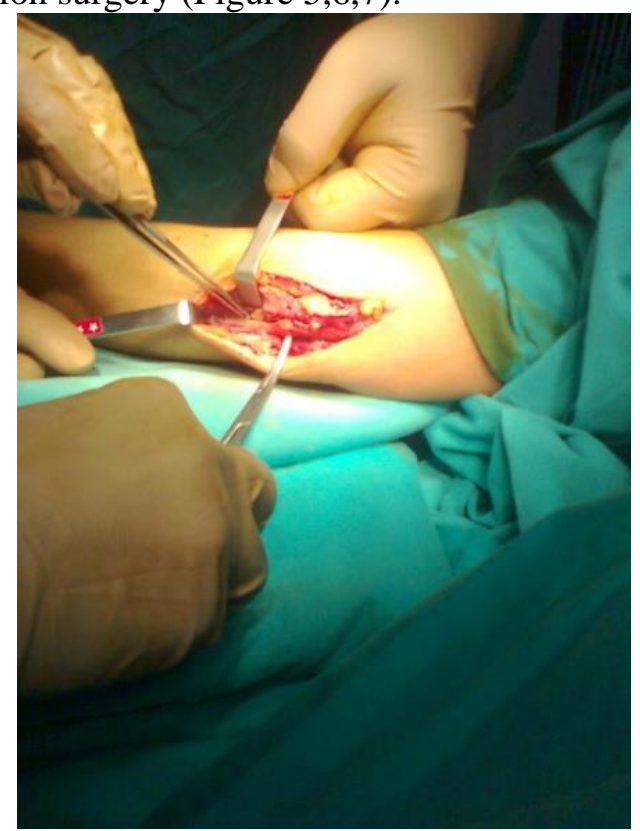

Figure 5: Release of ulnar nerve from cubital tunnel during anterior transposition (intra. Op.) 


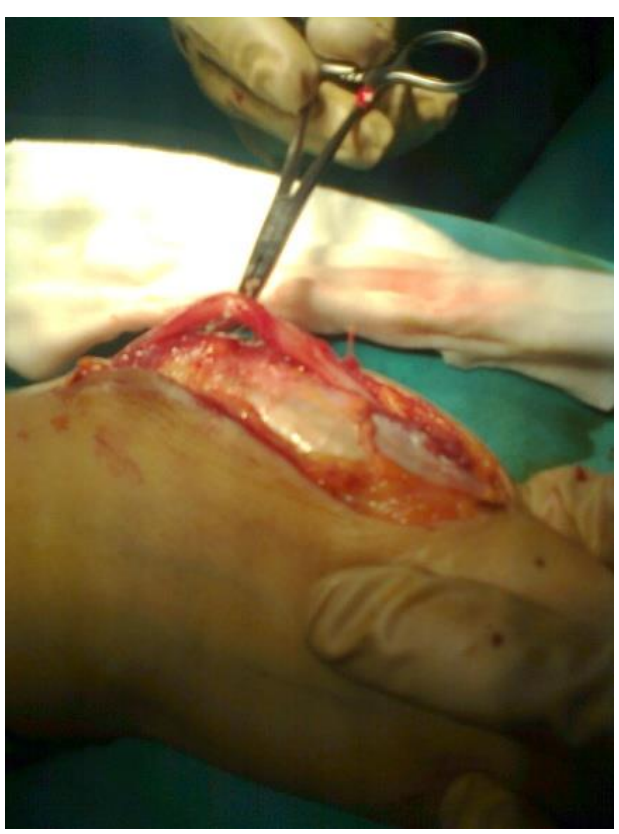

Figure 6: Sliding of ulnar nerve from the cubital tunnel to the during anterior transposition (intra op.)

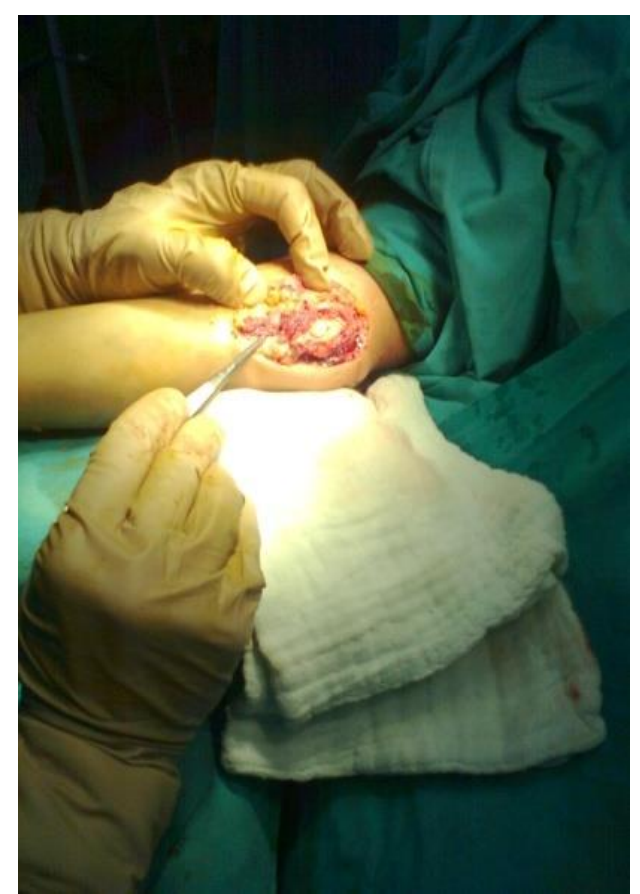

Figure 7: Subcutaneous and intramuscular transposition. Final intraoperative state

The patient was seen to fully open the ring finger and the claw to disappear completely in the postoperative 3rd month follow-up. The hypoesthesia at the hypothenar area decreased together with 50\% improvement in the extension limitation of the small finger (Figure 8).

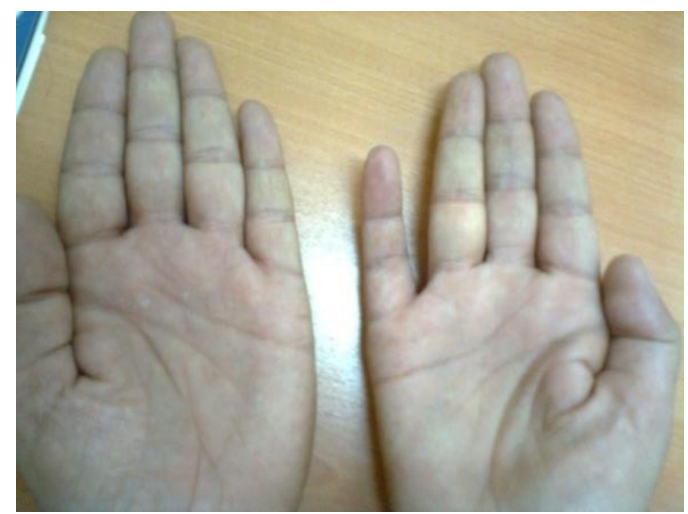

Figure 8: 3 months after Anterior Transposition the right $3^{\text {rd }}, 4^{\text {th }}$ and $5^{\text {th }}$ fingers can be extended at the interdigital joints.

The two point distinction test result was $25 \mathrm{~cm}$ pre-operatively and $3 \mathrm{~mm}$ after the second surgery. Tinel percussion and Flexion tests were completely negative at the right elbow. The Bishop evaluation system score was 7, indicating "a good level" of success. Improvement was observed in the postoperative 6th month EMG as well.

\section{Discussion}

Cubital Tunnel Syndrome is the second most common entrapment neuropathy of the upper extremities. The most important symptom can be pain and the symptoms can start insidiously and can be temporary at the beginning. Sensory disorders due to microvascular compression and neural ischemia occur at early stage in the hypothenar section of the hand, the small finger, and the ulnar half of the ring finger. If demyelination, axonal loss and motor symptoms occur with continuing compression or increased severity, these can lead to disorder of finger functions, dropping objects, and difficulties with catching and squeezing objects. Extension and adduction failure in small finger, and claw formation with the ring finger occur (Wartenberg sign). The Froment test is positive with weakness and atrophy in the interosseous muscles (lumbrical 3 and 4), adductor pollicis muscle and thehypothenar muscles. The Tinel percussion test and elbow flexion test are positive. Among objective tests, the two-point differentiation ( $<6 \mathrm{~mm}$ is normal) and pinprick tests are also positive (Dhia and Jaddue, 2009; Andertonand Webb, 2010; Thatte and 
Mansukhani, 2011). The Bishop score was evaluated over 12 points and the pre and postoperative results were compared (Asami, 2001; Dhia and Jaddue, 2009). Entrapment of ulnar nerve can develop in five anatomic locations: Struther's arcade, medial intermuscular septum, medial epicondyle, cubital tunnel, and deep flexor pronator aponeurosis. Specific tests as well as MRI, EMG, and high-resolution ultrasonography are used in clinical studies (Keineret al., 2009; Kutlay et al.,2009; Schnablet al., 2010; Iba et al.,2010; Thatte and Mansukhani, 2011; Kılıç and Özçakar, 2011). The Tinel percussion test, two-point differentiation test, Froment test, and Wartenberg sign were positive in our case. MRI and EMG were conducted. MRI revealed a supracondylar fracturerelated malunion and edema in the ulnar nerve route while EMG showed severe ulnar nerve entrapment.

The use of decompression techniques alone in cases with idiopathic cubital tunnel syndrome is preferred, primarily as they are less invasive with less possibility of complication development (Keiner et al.,2009; Flores,2010). The anterior transposition technique is one of the less invasive surgical procedures and is used in cases with secondary cubital tunnel neuropathy due to cubitus valgus deformity, an elbow flexion contracture of Grade 3according to the clinical classification of

McGowan and Dellon, significant weakness of the hand, or paralysis of interosseallumbrical muscles (Nikitinset al., 2002; Asamoto et al.,2005;Dhia and Jaddue , 2009;Keiner D et al.,2009;Flores 2010; Sreedbaran S et al.,2010). We first performed ulnar nerve decompression in our case. We planned decompression only so that the patient could recover quickly and return to work and social life in a short time with a smaller rate of complications. However, this treatment was not successful as seen in the follow-up and we then performed anterior transposition surgery for revision and used the subcutaneous technique proximally and the intramuscular technique distally. This approach is also supported with other studies where positive results were obtained (Dhia and Jaddue, 2009; Thatte and Mansukhani, 2011). Postoperative complete or near complete improvement was obtained with early intervention in our case with severe (Grade 3) entrapment neuropathy. However, improvement in symptoms has been reported to occur over a long period such as 2 to 5 years in delayed cases in the literature
(Keiner et al., 2009; Dhia and Jaddue, 2009; Anderton and Webb, 2010; Sreedbaran et al., 2010; Thatte and Mansukhani, 2011).The symptoms of our patient were found to have mostly improved during the five years of follow-up. These cases need long-term follow-up.

Ethics Committee Approval: Ethics committee approval was received for this study from Clinical Research Ethics Committee of: No Verbal Confirm of Patient

Author Contributions: İdea-EA ; Design TDA; Supervision- TDA; Funding-EA ; MaterialsEA,TDA ; Data Collection/Data Process-TDA.; Analyze or Comment-EA; Literature Scanning-EA; Writer of Paper-EA,TDA..

Conflict of Interest: No conflict of interest was declared by the authors.

Financial Disclosure: The authors declared that this study hasn't received any financial support.

\section{References}

Anderton M, Webb M, Cubital tunnel syndrome. British Journal of Hospital Medicine, November. 2010.Vol 71. No 11.

Asami A, The evaluation of pre and post operative classification system for cubital tunnel syndrome. HandSurgery, December 2001, Vol.6 No.2,187-190.

Asamoto S, Böker D.K, Jödicke A. Surgical treatment of ulnar nerve entrapment at the elbow. Neurol Med Chir (Tokyo) 2005:45(5);240-244:discussion 244-245.

Dhia A, Jaddue K, et al. Subcutaneous vs.submuscular ulnar nerve tranposition in moderate cubital tunnelsyndrome. The Open Orthopaedics Journal, 2009, Vol 3,78-82.

Flores L.P, Endoscopically assisted release of the ulnar nerve for cubital tunnel syndrome. ActaNeurochir.2010,152;619-625.

Iba K, Wada T, Tamakawa M et al. Diffusionweighted Magneticresonance imaging of the ulnar nerve in cubital tunnelsyndrome. Hand Surgery, 2010. Vol.15 No.1, 11-15. 
Keiner D, Gaab M.R, Schroeder H.W.S, Comparison of the long-term results of anterior transposition of the ulnar nerve or simple decompression in the treatment of cubital tunnel syndrome-aprospective study. ActaNeurochir.2009,151;311-316.

Kılıç E, Özçakar L, Ulnar nerve compression possibly due to aberrant veins:sonography is elucidatory for idiopathic cubital tunnel syndrome. RheumatolInt,2011,31;139-140.

Kutlay M, Çolak A, Şimşek $H$ et al. Use of ultrasonography in ulnar nerve entrapment surgery-a prospective study. NeurosurgRev ,2009, 12;225-232.

Nikitins M.D, Griffin P.A, Rice N. J, A dynamic anatomical study of ulnar nerve motion after anterior transposition for cubital tunnel syndrome. Hand Surgery,December 2002, Vol.7 No.2,177-182.

Schnabl S.M, Kisslinger F, Schramm A, etal. Objective outcome of partial medial epicondylectomy in cubital tunnel syndrome. Arch Orthop Trauma Surg. 2010,130;1549-1556

Sreedbaran S, Yam A.K.T, Tay S.C, Self-reported outcome following anterior transposition of ulnar nerve in the elderly. Hand Surgery, 2010, Vol 15 No 3,169-172.

Thatte M.R, MansukhaniK.A ,Compressive neuropathy in the upper limb.Indian Journal of Plastic Surgery, May-August, 2011, Vol 44 Issue 2.

Tubbs S.R, Loukas M, Apaydın N et al. Correlation between the lengths of the upper limb and cubital tunnel: potential use in patients with proximal ulnar nerve entrapment. SurgRadiolAnat, 2010,32;239-242.

Uysal M, Özkoç G, Tandoğan R et al. Combined unlar and carpal tunnel syndrome caused by pigmented villo-nodular tenosynovitis: a rare case. Arch Orthop Trauma Surg, 2007,127;563565. 